\title{
Osteogenic potential of graphene coated titanium is independent of transfer technique
}

DOI:

10.1016/j.mtla.2020.100604

\section{Document Version}

Accepted author manuscript

Link to publication record in Manchester Research Explorer

\section{Citation for published version (APA):}

Dubey, N., Morin, J. L. P., Luong-Van, E. K., Agarwalla, S. V., Silikas, N., Castro Neto, AH., \& Rosa, V. (2020). Osteogenic potential of graphene coated titanium is independent of transfer technique. Materialia, 9, [100604]. https://doi.org/10.1016/j.mtla.2020.100604

\section{Published in:}

Materialia

\section{Citing this paper}

Please note that where the full-text provided on Manchester Research Explorer is the Author Accepted Manuscript or Proof version this may differ from the final Published version. If citing, it is advised that you check and use the publisher's definitive version.

\section{General rights}

Copyright and moral rights for the publications made accessible in the Research Explorer are retained by the authors and/or other copyright owners and it is a condition of accessing publications that users recognise and abide by the legal requirements associated with these rights.

\section{Takedown policy}

If you believe that this document breaches copyright please refer to the University of Manchester's Takedown Procedures [http://man.ac.uk/04Y6Bo] or contact uml.scholarlycommunications@manchester.ac.uk providing relevant details, so we can investigate your claim.

\section{OPEN ACCESS}




\section{Journal Pre-proof}

Osteogenic potential of graphene coated titanium is independent of transfer technique

Nileshkumar Dubey, Julien Luc Paul Morin ,

Emma Kim Luong-Van , Shruti Vidhawan Agarwalla ,

Nikolaos Silikas, AH Castro Neto, Vinicius Rosa

PII:

S2589-1529(20)30021-1

DOI:

https://doi.org/10.1016/j.mtla.2020.100604

Reference:

MTLA 100604

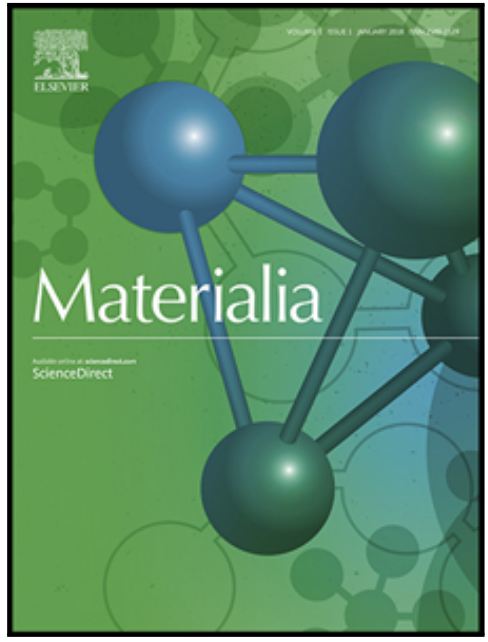

To appear in:

Materialia

Received date: $\quad 28$ November 2019

Accepted date: 27 January 2020

Please cite this article as: Nileshkumar Dubey, Julien Luc Paul Morin , Emma Kim Luong-Van , Shruti Vidhawan Agarwalla, Nikolaos Silikas, AH Castro Neto, Vinicius Rosa, Osteogenic potential of graphene coated titanium is independent of transfer technique, Materialia (2020), doi: https://doi.org/10.1016/j.mtla.2020.100604

This is a PDF file of an article that has undergone enhancements after acceptance, such as the addition of a cover page and metadata, and formatting for readability, but it is not yet the definitive version of record. This version will undergo additional copyediting, typesetting and review before it is published in its final form, but we are providing this version to give early visibility of the article. Please note that, during the production process, errors may be discovered which could affect the content, and all legal disclaimers that apply to the journal pertain.

(C) 2020 Acta Materialia Inc. Published by Elsevier Ltd. All rights reserved. 


\title{
Osteogenic potential of graphene coated titanium is independent of transfer technique
}

\author{
Nileshkumar $\underline{\text { Dubey }}{ }^{\mathrm{a}, \mathrm{b}} \quad \underline{\text { nileshd@umich.edu }}$ \\ Julien Luc Paul Morin ${ }^{c} \quad$ julien.morin@nus.edu.sg \\ Emma Kim Luong-Van ${ }^{c} \underline{\text { c2deklv@nus.edu.sg }}$ \\ Shruti Vidhawan $\underline{\text { Agarwalla }}^{a} \quad \underline{\text { shruti.va@u.nus.edu }}$ \\ Nikolaos Silikas $^{\mathrm{d}} \quad$ nikolaos.Silikas@manchester.ac.uk \\ AH Castro Neto $^{c} \quad \underline{\text { c2dhead@nus.edu.sg }}$ \\ Vinicius $\underline{R o s a}^{\mathrm{b}, c, \mathrm{e}, *} \quad$ denvr@nus.edu.sg
}

${ }^{a}$ School of Dentistry, University of Michigan. 1011 N University Ave, 48109, Ann Arbor, United States

${ }^{\mathrm{b}}$ Faculty of Dentistry, National University of Singapore, 9 Lower Kent Ridge Road, 119085, Singapore

${ }^{\mathrm{c}}$ Centre for Advanced 2D Materials and Graphene Research Centre, National University of Singapore, 6 Science Drive 2, 117546, Singapore

${ }^{\mathrm{d} B i o-A c t i v e ~ M a t e r i a l s ~ G r o u p, ~ D e p a r t m e n t ~ o f ~ M a t e r i a l s, ~ T h e ~ U n i v e r s i t y ~ o f ~ M a n c h e s t e r, ~ M 13 ~ 9 P L, ~}$ Manchester, United Kingdom

${ }^{\mathrm{e}}$ Department of Materials Science and Engineering, National University of Singapore, Blk EA, \#03-09 9 Engineering Drive 1, 117575, Singapore

*Corresponding author

V. Rosa (Vinicius Rosa)

denvr@nus.edu.sg

Faculty of Dentistry, National University of Singapore. 9 Lower Kent Ridge Road, 119085, Singapore 


\section{Graphical abstract}

\section{Wet transfer (WGp)}

Labor intensive

Technique sensitive

Water trapping

Long process (>17 h)

$\downarrow$ scalability, $\downarrow$ adoption

$\downarrow$ clinical translation potential
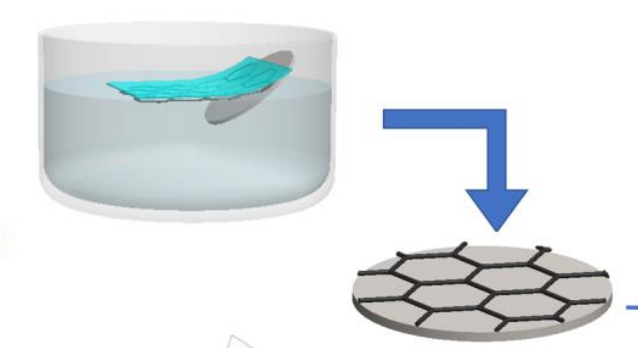

Increased gene expression
(vinculin, Talin, FAK, $\beta$ Integrin,

(vinculin, Talin, FAK, $\beta$ Integrin
Col1A1, Col2A1, Col3A1)

Dry transfer (DGp)

Mechanized

Reproducible

Liquid-free

Short process $(<3 \mathrm{~h})$

$\uparrow$ scalability, $\downarrow$ cost
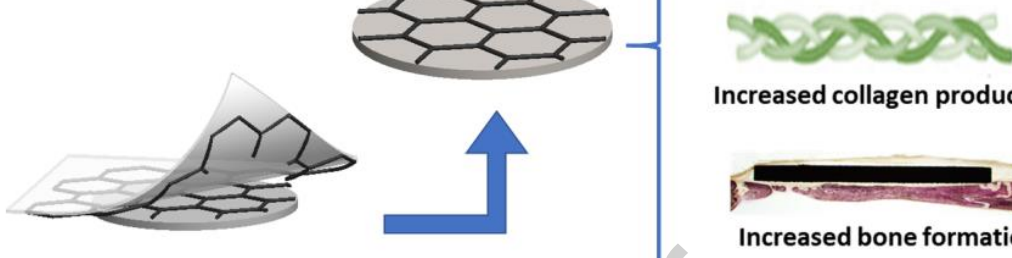

Increased collagen production

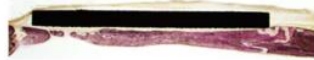

Increased bone formation on titanium

$\uparrow$ clinical translation potential 


\section{Abstract}

The rapid integration of titanium implants into native bone remains a challenge in orthopedics and dental implantology. Several surface modifications have been attempted, but with limited success. Graphene coating has emerged as a candidate for shortening integration time due to its osteogenic potential. The wet transfer technique (WGp) is widely used to demonstrate graphene's osteogenic potential but it is laborious and time-consuming, hence compromising industrial scalability and market adoption. Moreover, this transfer method traps water between the coating and the target substrate, which can further deteriorate the coating and compromise its clinical translation even more. Alternatively, the dry transfer (DGp) method, based on the mechanized application of pressurized heat, allows graphene films to be transferred directly from a tape onto titanium in few minutes at a low cost. Here, we show that a single dry transfer procedure can coat $>90 \%$ of titanium samples with graphene. Compared with uncoated titanium, DGp increased the expression of cellular adhesion and collagenrelated genes, collagen production, and bone formation. Raman and atomic force microscopy showed that cellular structures and stiffness were similar to those observed on uncoated titanium and X-ray powder diffraction confirmed that DGp favors the early formation of octacalcium phosphate. The results demonstrating the potential of DGp were similar to WGp, confirming that the changes in transfer procedures performed to improve the likelihood of industrial scalability and clinical translation do not compromise the osteogenic potential of the graphene coating on titanium.

Keywords: Osteointegration; implants; coating; osteogenesis; surface modification; differentiation; nanomaterial 


\section{Introduction}

As the world's population ages, the number of centenarians is expected to approach the 4 million mark by the year 2050 [1]. As individuals live longer, adopt more active lifestyles, and have increased access to health benefits, there is a rising demand for dental implants. Titanium and its alloys are used to fabricate dental and orthopedic implants, thanks to the combination of their high mechanical strength, chemical stability, biocompatibility, and osteointegration. Specifically, osteointegration refers to the functional connection between living bone and the surface of a load-carrying implant, which can take up to six months to be fully effective [2]. In a previous study, $21 \%$ of patients cited the long healing time as a negative factor, which can result in low adherence to rehabilitation [3]. Systemic conditions leading to low bone density (osteoporosis, diabetes, or radiotherapy) also pose challenges for titanium implants [4].

Several surface treatments have been attempted to enhance mineralization and ensure rapid and longlasting osteointegration of titanium implants [5]. For instance, hydroxyapatite can increase bone formation in vivo [6], but its poor mechanical properties make it prone to delamination from the implant [7]. Likewise, coatings functionalized with bone morphogenetic protein (BMP) or containing viral vectors expressing BMP-7 can also modulate osteogenic differentiation [8]. However, growth factors are costly, and viral vectors may cause immunological rejection and disrupt normal gene functions [9]. Hence, the development of cost-effective strategies that favor bone formation on implants without disrupting normal function is of great interest.

Graphene has emerged as a promising material to improve the properties of titanium, corrosion resistance [10], protection against biofilm formation, and osteointegration potential [11, 12].

Atomically-thin graphene films are cytocompatible and can be transferred onto stainless steel, titanium, and full-sized dental implants with more than $90 \%$ coverage yield [11, 13, 14]. Moreover, graphene has been shown to elevate expression of several osteogenic genes and proteins, such as alkaline phosphatase (ALP), runt-related transcription factor 2 (RUNX2), osteopontin (OPN), and osteocalcin (OCN); it also augments mineralization potential even in the absence of osteogenic inducers [15-17]]. A recent study showed that titanium coated with graphene via a wet transfer technique (WGp) increased bone formation in vivo [18]. Regardless of its promising osteogenic potential, it is still not known whether the biological processes leading to matrix secretion and its composition on graphene are similar to those observed on bare titanium.

Despite the achievements observed with WGp, there are important limitations inherent in the wet transfer technique. Firstly, it is very labor-intensive and time-consuming $[11,18]$. Moreover, as it is mostly performed manually, it suffers from high sample and batch-to-batch variation, depending on the operator's skill. It also requires the use of polymethyl methacrylate (PMMA) as an intermediate polymer, which is well-known for inducing cracks on graphene [19]. Furthermore, the high molecular weight and viscosity of PMMA make it difficult to remove with acetone, inevitably affecting graphene's mechanical properties $[20,21]$. The annealing step required to remove the PMMA further increases the time and cost to produce the graphene coating. Finally, the WGp often traps water between the target substrate and the graphene film, which, upon evaporation, creates large folds and additional cracked 
areas that compromise the long-term integrity and quality of the coating $[22,23]$. Although graphene coatings via WGp are interesting from a purely scientific perspective, they are unlikely to become a clinical reality due to the inherent limitations of this transfer technique and its low potential for industrial scalability.

To overcome the problems associated with the presence of a liquid phase, for industrial scalability, new dry transfer techniques (DGp) have been developed. For instance, the roll-to-roll technique allows a clean transfer of graphene on a large scale with potential economic advantages [24]. The vacuumassisted dry transfer and hot-press techniques rely on pressure gradients to deposit high-quality graphene from a transfer tape onto the target substrate without using hazardous chemicals [11, 14]. Likewise, procedures such as electrochemical delamination allow for the detachment of graphene from copper without hazardous chemicals [13]. Hence, dry transfer methods could increase yield through fast and clean deposition of large areas of graphene in a short time, bringing graphene-coated titanium closer to clinical reality.

The objective of this study was to characterize the in vitro and in vivo osteogenic potential of graphenecoated titanium via dry transfer and to benchmark its biological outcomes against uncoated and graphene-coated titanium via WGp.

\section{Experimental}

\subsection{Sample and graphene production, transfer and characterization}

Titanium discs (Ti-6Al-4V grade 4, $12.7 \mathrm{~mm}$ diameter, $1 \mathrm{~mm}$ thickness, Vulcanium, USA) were polished with SiC paper (up to 2500, 150 rpm, 20 N, EcoMet 30, Buehler, Germany), and washed in an ultrasonic bath with acetone and deionized water (30 min each).

Graphene was grown by a single operator using a chemical vapour deposition (CVD) custom-built furnace in a Class 1000 cleanroom facility. Briefly, the copper foil (Graphene Platform, Tokyo, Japan) was placed in a quartz tube ( 30 mTorr) filled with hydrogen gas $(8 \mathrm{sccm})$. Thereafter, the copper foil was annealed at $1010^{\circ} \mathrm{C}$ at 3 Torr for $35 \mathrm{~min}$. The graphene film was grown with the pyrolysis of methane (16 sccm, 30 min at 500 mTorr). Thereafter, the tube was cooled to room temperature in $\mathrm{H}_{2}$ atmosphere.

The graphene film was transferred onto both sides of the titanium discs using either wet or dry transfer technique. For the wet transfer technique (WGp), the graphene on copper foil was coated with a $60 \mu \mathrm{m}$ PMMA layer and cured $\left(180^{\circ} \mathrm{C}, 10 \mathrm{~min}\right)$. The uncoated surface of the copper foil was etched with oxygen plasma ( $50 \mathrm{sccm}, 50 \mathrm{~W}, 5 \mathrm{~min}$, FEMTO Science Inc, Korea). The copper substrate was etched for 8 $\mathrm{h}$ with $1.5 \%$ ammonium persulphate solution (APS) and the resulting graphene/PMMA set cleaned in deionized water for $2 \mathrm{~h}$. Thereafter, the graphene/PMMA was scooped with a disc from the water following by the removal of the PMMA with acetone $(12 \mathrm{~h})$ followed by cleaning with IPA and deionized water ( $3 \mathrm{~h}$ and $30 \mathrm{~min}$, respectively). 
For the dry transfer (DGp), the graphene film on copper foil was coated with polydimethylsiloxane (PDMS 10:1, Sylgard 184, Dow Corning, USA) and set at $135^{\circ} \mathrm{C}$ for $20 \mathrm{~min}$. Afterward, the uncoated side was etched with oxygen plasma and the copper foil was removed with APS The graphene/PDMS tape was washed with deionized water $(2 \mathrm{~h})$ and dried $\left(10 \mathrm{~min}, 100^{\circ} \mathrm{C}\right)$. The tape was placed on the disc under uniaxial pressure $\left(150 \mathrm{~N}, 4 \mathrm{~min}\right.$ at $\left.150^{\circ} \mathrm{C}\right)$. Finally, the PDMS was peeled off leaving the graphene on the disc surface.

Ten samples of each group were characterized by Raman spectroscopy (Raman Microscope CRM 200, Witec, Germany) and atomic force microscope (AFM, Bruker AXS, Germany). Ten random areas were mapped per sample for the identification of $G\left(\sim 1580 \mathrm{~cm}^{-1}\right), D\left(\sim 1354 \mathrm{~cm}^{-1}\right)$ and 2D peaks $(2680 \mathrm{~cm}$ $\left.{ }^{1}\right)$. Surface topography was analysed using tapping mode AFM with a silicon nitride tip (resonance frequency: $40-90 \mathrm{kHz}$, spring constant: $0.4 \mathrm{~N} / \mathrm{m}$ ).

\subsection{Orthotopic bone formation in vivo}

All animal experiments were approved by the Office of Institutional Animal Care \& Use Committee (NUS-R16-0344). Thirty-six Sprague-Dawley rats (male, NTac:SD, 6 weeks, 220-280 g, InVivos, Singapore) were anesthetized and a critical-sized calvarial defect created with a trephine. The discs were placed into the defect and the incision was closed with resorbable sutures. Each group was comprised of six samples for each time point.

The bone formation was evaluated in live animals at 4- and 8-weeks post-implantation using micro-computed tomography $(\mu \mathrm{CT})$ imaging system (90 kV, 200 mA, R-mCT, Rigaku, Japan). Threedimensional images were reconstructed using the Mimics software (Materialise, Belgium) and the percentage of bone volume/total volume (BV/TV) was calculated.

After being scanned, the animais were sacrificed and the skulls fixed for $24 \mathrm{~h}$ with $10 \%$ buffered formalin solution, dehydrated in alcohol series and embedded with methyl methacrylate. The resin block was cut (Exkat 310 Band Cutting Exakt, Norderstedt, Germany) and serial cross-sections were prepared (150 $\mu \mathrm{m}$ thickness). The sections were bonded to plexiglass, polished to a thickness of $60-100$ microns (Exakt 400 Micro Grinding System) and stained for hematoxylin and eosin.

\subsection{In vitro evaluation of extracellular matrix deposition and adhesion molecules}

For all assays described in this section, MG-63 were seeded at an initial density of $2 \times 10^{4}$ cells per disc and cultured under basal growth media (DMEM and $10 \%$ fetal bovine serum, Invitrogen) up to 5 days. The relative gene expression of the collagen types I alpha 1 (COL) 1, 2 and 3, and focal adhesion genes vinculin, focal adhesion kinase (FAK), $\beta$-integrin and talin were quantified by qPCR (gene sequences in Supplementary Information Table 1). At each time point, cells were detached (Trypsin, Invitrogen, USA), total RNA was isolated (Purelink RNA Mini Kit, Invitrogen) and cDNA synthesis performed (iScript RT Supermix, Bio-Rad, USA). Genetic expressions were analyzed by quantitative real-time QPCR (ITaqMan system and CFX Connect Real-Time System, Bio-Rad). Data were analysed using the $\triangle \Delta C T$ method and normalized against GAPDH.

The total collagen production was measured from the culture media and cell lysate (Sircol, Biocolor, Carrickfergus, United Kingdom) using type I collagen as a standard. This method is based on the selective 
binding property of the Sirius Red dye to the [Gly-X-y] tripeptide end sequence of mammalian collagen. The total amount of collagen production was normalized against DNA content (DNAzol, Invitrogen) obtained from separated cultures.

The cellular elastic modulus was measured at the endpoint ( 5 days) using NanoWizard AFM (JPK Instruments, Berlin, Germany), mounted on a modified microscope stage of an upright optical microscope $\left(512 \times 512\right.$ pixels $^{2}$ at line rates of $0.7 \mathrm{~Hz}$ Axiovert 200, Carl Zeiss, Microlmaging, Germany). The force curves were obtained in contact mode in liquid using silicon nitride cantilevers (MLCT-Microlever Probes, Veeco Instruments, Germany) with constant force at $20 \mathrm{mN} / \mathrm{m}$. Ten cells per group were indented at 4 random locations.

The extracellular matrix was characterized by Raman spectroscopy and X-ray diffraction (XRD). For Raman, at each time point, cells washed with phosphate-buffered saline (Invitrogen) and fixed with 4\% paraformaldehyde for $10 \mathrm{~min}$ at room temperature. Thereafter, cells were washed in PBS and the Raman spectra (Raman Microscope CRM 200, Witec, Germany) were obtained at $532 \mathrm{~nm}$ with 100X objective. The spectra ( 2 accumulations of $90 \mathrm{~s}$ exposure) were acquired from 20 cells from each group. For the XRD analysis, cells were rinsed three times with double-distilled water $\left(\mathrm{ddH}_{2} \mathrm{O}, \mathrm{pH} 8.0\right.$, resistivity $18.2 \mathrm{M} \Omega \mathrm{cm}$, Simplicity Purification System, Millipore, USA), scraped with a plastic spatula and vortexed for $5 \mathrm{~min}$ in 1.3\% sodium hypochlorite (Acros Organics, USA) to remove organic components. Crystals were pelleted by centrifugation, washed three times in $\mathrm{dd}_{2} \mathrm{O}$, dehydrated with an increasing gradient of ethanol and freeze-dried overnight. The crystallographic analysis was performed using a D8 Advance Powder X-ray Diffractometer (Bruker AXS, Germany) with a Ni filter and CuKa radiation $\left(\lambda=1.5425 \mathrm{~A}^{\circ}\right.$ ) at $40 \mathrm{kV}$ and $40 \mathrm{~mA}$ (scan range: $10-60^{\circ}$, scanning rate of $0.02^{\circ} /$ second).

\subsection{Statistical analysis}

All tests were performed thrice in three independent triplicates unless stated otherwise. Shapiro-Wilk and Lavene's tests were performed for checking normality and homogeneity. One-way ANOVA and Tukey's test was used to analyse the data from BV/TV and the other tests were analysed with MannWhitney test (SPSS, USA). A $5 \%$ pre-set significance level was set for all the analysis.

\section{Results}

\subsection{Sample characterization}

Fig. 1 shows the morphology and the Raman characterization for the uncoated (Control) and graphenecoated samples via the wet (WGp) and dry (DGp) techniques. The AFM (Fig. 1A) shows that the graphene films conformed to the surface topography of the discs. Additionally, there were no clear differences in the surface features between WGp and DGp. The Raman characterization revealed that DGp coated approximately $90 \%$ of the sample, whereas WGp fully coated it (Fig. 1B). However, the ID/IG ratio (indicative of defects) was fairly similar in both transfer techniques ( 0.47 for WGp and 0.64 for DGp). The full width at half maximum (FWHM) of the 2D peak for DGp was greater than $30 \mathrm{~cm}^{-1}$, suggesting possible folding and bundling in some areas (Table 1).

\subsection{Effects of graphene transfer technique on osteogenic potential in vivo}


Fig. 2 shows the histological and tomographic outcomes 4 and 8 weeks after the implantation of the uncoated and graphene-coated samples. Fig. 2A shows that both WGp and DGp induced higher extension of the bone margins to the center of the discs at 4 weeks. After 8 weeks, there was a significantly larger amount of bone in the lower surface of WGp and DGp discs compared with the Control. This phenomenon can be seen in Fig. 2B, where the Control disc can be identified in the inner part of the skull even after 8 weeks, while the graphene-coated specimens are already barely visible at 4 weeks. Fig. $2 \mathrm{C}$ shows complete gap closure after WGp and DGp, whereas a defective area is still present in the Control (arrow). Finally, the bone volume/total volume (BV/TV) ratio was significantly higher in samples coated with graphene via both techniques compared to the Control at both time points. At Week 8, the BV/TV ratio was 19.0 for the Control, 32.7 for WGp and 31.2 for DGp (Fig. 2D).

\subsection{Effects of graphene transfer technique on gene expression, collagen production and extracellular matrix characteristics}

The impact of graphene coatings and their transfer techniques on the biological outcomes of MG-63 cells was subsequently investigated. To this end, cells were seeded on graphene-coated and uncoated samples and cultured in basal growth media for up to 5 days. Firstly, the expression of genes activated by cellular mechanical stimulation was assessed. Both WGP and DGp promoted a significant increase in the genetic expression of vinculin, talin, focl adhesion kinase (FAK), and $\beta$-integrin at all time points tested compared with the Control (Fig. 3). Despite the statistical differences observed on Days 1 and 3 for vinculin and talin, the overall expressions obtained from both WGp and DGp were similar.

Subsequently, the expression of collagen-related genes was assessed (Figs. 4A-C). A significant increase in the expression of all genes in graphene-coated samples was observed compared with the Control (except for COL1A1, Day 1). The total collagen produced per $\mu \mathrm{g}$ of DNA was also significantly higher for WGp and DGp at all time points compared with the Control (Fig. 4D). In terms of cell stiffness, measured by AFM at the endpoint, there was no significant difference in the Young modulus obtained from cells among the three groups (Fig. 4E).

To confirm that cell structures and mineralization on graphene were similar to those observed on unmodified titanium, we characterized the cultures after 5 days. The Raman characterizations showed spectra obtained in the range of 900 to $1800 \mathrm{~cm}^{-1}$. Overall, the fingerprints obtained from the three groups exhibited similar patterns at all time points. The bands were at 1239 and $1318 \mathrm{~cm}^{-1}$, which correspond to amide III, and at $1660 \mathrm{~cm}^{-1}$, which corresponds to amide I. The fingerprint of $\mathrm{CH} 2$-wag from collagen was also observed at $1450 \mathrm{~cm}^{-1}$. A prominent phenylalanine band representing the organic matrix of bone was observed at $1003 \mathrm{~cm}^{-1}$. The spectral region related to $\mathrm{V3PO} 43-$ was detected at $1030 \mathrm{~cm}^{-1}$, and weakly between 948 and $970 \mathrm{~cm}^{-1}$. To investigate levels of the transient mineral phase during osteogenic differentiation, we magnified and deconvoluted the phosphate spectral region between 948 and $970 \mathrm{~cm}^{-1}$ (Supplementary Information). A decrease in the region between 945 and 952 $\mathrm{cm}^{-1}$, corresponding to amorphous calcium phosphate for both WGp and DGp, was observed on Day 5 . The region corresponding to octacalcium phosphate (OCP) at $958 \mathrm{~cm}^{-1}$ was identifiable for both WGp and DGp on Day 3 and at a lower level for the Control only at the endpoint. The crystal structure of the mineral content was assessed by X-ray diffraction (XRD), and the $2 \theta$ positions matched those reported 
by the Joint Committee on Powder Diffraction Standards (JCPDS). As shown in Fig. 5 , the cell culture mineral exhibited a single high peak in the $2 \theta$ at a range between $30.5^{\circ}$ and $34.0^{\circ}$, and centered at $31.8^{\circ}$, which is characteristic of a poorly crystallized apatite known as calcium deficient hydroxyapatite (CDHA). Other intense reflections of apatite material were identified as amorphous calcium phosphate (ACP), tricalcium phosphate (TCP), and dicalcium phosphate anhydrous (DCPA) at 28.0 $0^{\circ}, 45.6^{\circ}$, and $55.9^{\circ}$, respectively. Crystalline octacalcium phosphate (OCP) peak was visible at $26^{\circ}$ on Day 5 for WGp and DGp, while it was absent in the Control.

\section{Discussion}

Titanium and its alloys are used for the fabrication of biomedical and dental implants, but the amount of time required for their osteointegration is a crucial factor for their success. Graphene coating has emerged as a candidate to reduce that time. In spite of its osteogenic potential, WGp is unlikely to become a clinical reality due to the critical limitations inherent in the transfer process. Moreover, little is known about the biological events and the nature of mineralization on graphene. The elucidation of the osteogenic potential and mineralization character of a graphene coating deposited on titanium via a scalable DGp technique is crucial for the translation of this coating into clinical practice.

\subsection{Effects of transfer technique on osteogenesis}

DGp coated approximately $90 \%$ of the titanium surface with one transfer procedure. Although it left $10 \%$ of the surface uncoated, this can potentially be solved with an extra transfer procedure. This strategy has been proven capable of increasing the coverage yield from $82 \%$ to $99 \%$ with a dry transfer method [11]. It must be emphasized that a second transfer procedure to increase coverage yield via the dry technique is advantageous in terms of time as it only takes a few minutes, compared to the several hours required in WGp.

First, we assessed the osteogenic potential of DGp and benchmarked it against uncoated titanium (Control) and graphene-coated titanium via WGp. A previous study that evaluated bone formation around Ti-6Al-4V implants in the femoral condyle of rabbits in a period of 24 weeks reported a higher $\mathrm{BV} / \mathrm{TV}$ ratio on implants coated with graphene via the wet transfer technique $(60 \%)$ compared with the Control (43\%) [18]. A similar trend was observed in this study's graphene-coated implants, where a thicker bone layer was formed (Fig. 2A), and the BV/TV ratio was significantly higher compared with the Control (Fig. 2D). Notably, there was no difference in the BV/TV ratio between WGp and DGp, suggesting that the in vivo osteogenic potential is independent of transfer technique.

\subsection{Effects of transfer technique on extracellular matrix and mineralization and characteristics}

Graphene is known to induce osteogenic differentiation without the use of chemical inducers $[15,16]$. This process has been linked to the activation of genes and the integrin-FAK axis that is likely mechanostimulated by the surface features of graphene [15-17]. Hence, we investigated whether the transfer technique could affect the expression of genes related to sensing mechanical stimuli. Overall, 
graphene induced significantly higher expression of the genes assessed for all the tested time points compared with the Control (Fig. 3). Vinculin is the key regulator of focal adhesion and interacts with talin and FAK to mediate integrin linkage to the actin cytoskeleton $[25,26]$. Likewise, talin binds to the $\beta$ integrin subunits and has been suggested to function as a mechanosensitive signaling hub [27]. With the exception of vinculin and talin on Day 1, there were no differences in the genetic expression profiles between WGp and DGp. This may indicate that the transfer technique is irrelevant to the stimuli provided from graphene surface features and their interaction with cell membranes.

The extracellular matrix (ECM) deposited by osteoblast lineages has an intricate composition of collagenous and non-collagenous proteins and calcium phosphates [28]. The ECM is connected to Factin cytoskeleton by the integrin [29] and the interaction between ECM and collagen promotes mineralization [30]. Approximately $51 \%$ of the bone in the vicinity of implants loaded for 6 months is composed of transverse and longitudinal collagen fibers [31]. Additionally, MG-63 has higher ALP and OCN gene expression potential when the surface roughness of titanium is optimized by a few micrometers [32]. As graphene provides a wrinkled surface to titanium and changes its arithmetic roughness average $\left(R_{a}\right)$ only at the nanometer scale [11], we investigated whether the anchor points provided by nanocoating to cells could increase their potential for collagen production. To this end, we first assessed the expression of collagen genes and observed significant increases when cells were cultured on graphene-coated samples compared with the Control (Fig. s 4A-C). Likewise, mesenchymal stem cells cultured in basal growth media on graphene-coated substrates presented increased gene and protein expression of COL-1 [15, 33]. Collagen adsorbed on implants has been shown to provide the framework for matrix mineralization and to promote the osteointegration of titanium implants [34]. Remarkably, graphene-coated titanium increased the total collagen production by at least 28,40 , and $35 \%$, respectively, at each time point (Fig. 4D). This enhancement could provide increased bone formation on graphene-coated samples in vivo (Fig. 2).

For safe clinical translation, the osteogenic potential of graphene should ideally not lead to gross cell alteration. The Raman analysis revealed that the spectra for the cell structures were similar in the Control, WGp, and DGp groups at all time points evaluated (Fig. 5). A similar trend was observed in cell stiffness at the endpoint (Fig. 4B). We also characterized the mineralized matrix. Although several studies have reported the mineralization potential of graphene $[12,33]$, there is no evidence that the mineral structures on graphene-coated titanium resemble those on unmodified titanium. Overall, the XRD spectra showed a similar pattern in all three groups, with a wideband (approximately $16-25^{\circ}$ ), suggestive of the amorphous phase (e.g., collagen), and peaks related to mineralization (Fig. 5). Amorphous calcium phosphate is the first insoluble phase which precipitates the formation of stable crystalline phases, such as octacalcium phosphate (OCP) or apatitic products [35]. Both graphene-coated samples presented peaks at $26^{\circ}$ that are suggestive of the presence of OCP, which is a marker for the onset of mineralization and a precursor phase of hydroxyapatite [36].

Despite these promising results, this study has some limitations. Firstly, the calvarial defect model does not provide the mechanical stress which implants are often subjected to. Nevertheless, this model has been extensively used to characterize the osteogenic potential of novel materials. Also, the MG-63 cells are arrested in a pre-osteoblast state and can exhibit variation in their mineralization potential. 
However, this cell line offers an unlimited number of cells with low passage variability and an integrin subunit profile similar to that of human ones [37]. Future studies should evaluate the osteogenic potential of graphene nanocoating on implants subjected to functional loading and other relevant clinical aspects, such as the coating's long-term stability on titanium.

\section{Conclusion}

Graphene has osteogenic potential and is envisioned as a promising coating to enhance the osseointegration of titanium implants. WGp is the most explored method to deposit graphene on titanium but has intrinsic limitations that limit its industrial scalability and clinical translation. Alternative methods, such as DGp, are less laborious and more scalable, but their osteogenic potential in vivo remains largely unknown. Overall, in this study, the graphene-coated samples exhibited greater bone formation and higher expression of genes involved in such biological processes compared with the uncoated samples. Most importantly, DGp exhibited osteogenic potential, gene expression, and collagen production comparable to WGp, and both presented similar spectra for mineralization and cellular fingerprints to those observed in the Control, suggesting that the coating does not harm cell structures or induce abnormal mineralization. Therefore, our results suggest that DGp can be a promising coating with industrial scalability and potential for clinical translation to promote the integration of titanium into orthopedic and dental applications.

\section{Declaration of Competing Interest}

The authors declare that they have no known competing financial interests or personal relationships that could have appeared to influence the work reported in this paper.

\section{Acknowledgements}

This research was supported by a grant from the National University Health System, Singapore (NUHSRO/2016/132/NUHS O-CRG Oct/25, R-221-000-109-733) and Singapore Ministry of Education, Singapore (Academic Research Fund Tier 1, R-221-000-132-114). 


\section{References}

[1] United Nations, World Population Prospects - 2015 Revision. Available at https://population.un.org/wpp/Publications/Files/WPP2015 DataBooklet.pdf. Accessed on Nov 22, 2019., 2015.

[2] S. Ersanli, C. Karabuda, F. Beck, B. Leblebicioglu, Resonance frequency analysis of one-stage dental implant stability during the osseointegration period, J. Periodontol., 76 (2005), pp. 1066-71, 10.1902/jop.2005.76.7.1066

[3] A. Johannsen, U. Wikesjo, G. Tellefsen, G. Johannsen, Patient attitudes and expectations of dental implant treatment--a questionnaire study, Swed. Dent. J., 36 (2012), pp. 7-14,

[4] M.M. Bornstein, N. Cionca, A. Mombelli, Systemic conditions and treatments as risks for implant therapy, Int. J. Oral Maxillofac. Implants, 24 (2009), pp. 12-27,

[5] F. Rupp, L. Liang, J. Geis-Gerstorfer, L. Scheideler, F. Hüttig, Surface characteristics of dental implants: A review, Dent. Mater., 34 (2018), pp. 40-57, 10.1016/j.dental.2017.09.007

[6] A. Tonetto, P.W. Lago, M. Borba, V. Rosa, Effects of chrondro-osseous regenerative compound associated with local treatments in the regeneration of bone defects around implants: an in vivo study, Clin. Oral Investig., 20 (2016), pp. 267-74, 10.1007/s00784-015-1509-1

[7] J.P. Collier, V.A. Surprenant, M.B. Mayor, M. Wrona, R.E. Jensen, H.P. Surprenant, Loss of hydroxyapatite coating on retrieved, total hip components, J. Arthroplasty, 8 (1993), pp. 389-93, [8] S. Chen, J. Yang, H. Wang, Y. Chao, C. Zhang, J. Shen, P. Zhang, Adenovirus encoding BMP-7 immobilized on titanium surface exhibits local delivery ability and regulates osteoblast differentiation in vitro, Arch. Oral Biol., 58 (2013), pp. 1225-1231, 10.1016/j.archoralbio.2013.03.019

[9] P.D. Robbins, H. Tahara, S.C. Ghivizzani, Viral vectors for gene therapy, Trends Biotechnol., 16 (1998), pp. 35-40, 10.1016/s0167-7799(97)01137-2

[10] R. Malhotra, Y.M. Han, J.L.P. Morin, E.K. Luong-Van, R.J.J. Chew, A.H. Castro Neto, C.A. Nijhuis, V. Rosa, Inhibiting Corrosion of Biomedical-Grade Ti-6Al-4V Alloys with Graphene Nanocoating, J. Dent. Res., (2020), pp., 10.1177/0022034519897003

[11] S.V. Agarwalla, K. Ellepola, M.C.F.d. Costa, G.J.M. Fechine, J.L.P. Morin, A.H. Castro Neto, C.J. Seneviratne, V. Rosa, Hydrophobicity of graphene as a driving force for inhibiting biofilm formation of pathogenic bacteria and fungi, Dent. Mater., 35 (2019), pp. 403-413, 10.1016/j.dental.2018.09.016 [12] N. Dubey, K. Ellepola, F.E. Decroix, J.L. Morin, A. Castro Neto, C.J. Seneviratne, V. Rosa, Graphene onto medical grade titanium: an atom-thick multimodal coating that promotes osteoblast maturation and inhibits biofilm formation from distinct species, Nanotoxicology, 12 (2018), pp. 274-289,

[13] J.L.P. Morin, N. Dubey, F.E.D. Decroix, E.K. Luong-Van, A.H. Castro Neto, V. Rosa, Graphene transfer to 3-dimensional surfaces: a vacuum-assisted dry transfer method, 2D Materials, 4 (2017), pp. 025060, 10.1088/2053-1583/aa6530

[14] C.L.C. Rodriguez, F. Kessler, N. Dubey, V. Rosa, G.J.M. Fechine, CVD graphene transfer procedure to the surface of stainless steel for stem cell proliferation, Surf. Coat. Technol., 311 (2017), pp. 10-18, 10.1016/j.surfcoat.2016.12.111

[15] H. Xie, T. Cao, J.V. Gomes, A.H. Castro Neto, V. Rosa, Two and three-dimensional graphene substrates to magnify osteogenic differentiation of periodontal ligament stem cells, Carbon, 93 (2015), pp. 266-275, http://dx.doi.org/10.1016/i.carbon.2015.05.071

[16] T.R. Nayak, H. Andersen, V.S. Makam, C. Khaw, S. Bae, X. Xu, P.L. Ee, J.H. Ahn, B.H. Hong, G. Pastorin, B. Ozyilmaz, Graphene for controlled and accelerated osteogenic differentiation of human mesenchymal stem cells, ACS Nano, 5 (2011), pp. 4670-8, 10.1021/nn200500h 
[17] H. Xie, T. Cao, A. Franco-Obregón, V. Rosa, Graphene-Induced Osteogenic Differentiation Is Mediated by the Integrin/FAK Axis, Int. J. Mol. Sci., 20 (2019), pp., 10.3390/ijms20030574

[18] K. Li, C. Wang, J. Yan, Q. Zhang, B. Dang, Z. Wang, Y. Yao, K. Lin, Z. Guo, L. Bi, Evaluation of the osteogenesis and osseointegration of titanium alloys coated with graphene: an in vivo study, Sci. Rep., 8 (2018), pp. 1843,

[19] L.G.P. Martins, Y. Song, T. Zeng, M.S. Dresselhaus, J. Kong, P.T. Araujo, Direct transfer of graphene onto flexible substrates, Proceedings of the National Academy of Sciences, 110 (2013), pp. 17762-17767, 10.1073/pnas.1306508110

[20] W. Choi, M.A. Shehzad, S. Park, Y. Seo, Influence of removing PMMA residues on surface of CVD graphene using a contact-mode atomic force microscope, RSC Advances, 7 (2017), pp. 6943-6949, $10.1039 / c 6 r a 27436 f$

[21] Y. Chen, X.-L. Gong, J.-G. Gai, Progress and Challenges in Transfer of Large-Area Graphene Films, Advanced Science, 3 (2016), pp., 10.1002/advs.201500343

[22] X. Liang, B.A. Sperling, I. Calizo, G. Cheng, C.A. Hacker, Q. Zhang, Y. Obeng, K. Yan, H. Peng, Q. Li, X. Zhu, H. Yuan, A.R. Walker, Z. Liu, L.M. Peng, C.A. Richter, Toward clean and crackless transfer of graphene, Acs Nano, 5 (2011), pp. 9144-53, 10.1021/nn203377t

[23] X. Li, Y. Zhu, W. Cai, M. Borysiak, B. Han, D. Chen, R.D. Piner, L. Colombo, R.S. Ruoff, Transfer of Large-Area Graphene Films for High-Performance Transparent Conductive Electrodes, Nano Lett., 9 (2009), pp. 4359-4363, 10.1021/nl902623y

[24] H. Xin, W. Li, A review on high throughput roll-to-roll manufacturing of chemical vapor deposition graphene, Applied Physics Reviews, 5 (2018), pp., 10.1063/1.5035295

[25] J.D. Humphries, P. Wang, C. Streuli, B. Geiger, M.J. Humphries, C. Ballestrem, Vinculin controls focal adhesion formation by direct interactions with talin and actin, The Journal of cell biology, 179 (2007), pp. 1043-1057,

[26] P. Atherton, B. Stutchbury, D. Jethwa, C. Ballestrem, Mechanosensitive components of integrin adhesions: Role of vinculin, Exp. Cell Res., 343 (2016), pp. 21-27,

[27] B.T. Goult, J. Yan, M.A. Schwartz, Talin as a mechanosensitive signaling hub, The Journal of Cell Biology, 217 (2018), pp. 3776-3784, 10.1083/jcb.201808061

[28] A.I. Alford, K.M. Kozloff, K.D. Hankenson, Extracellular matrix networks in bone remodelling, The International Journal of Biochemistry \& Cell Biology, 65 (2015), pp. 20-31, 10.1016/j.biocel.2015.05.008

[29] Z. Sun, S.S. Guo, R. Fässler, Integrin-mediated mechanotransduction, The Journal of Cell Biology, 215 (2016), pp. 445-456, 10.1083/jcb.201609037

[30] G.B. Schneider, R. Zaharias, C. Stanford, Osteoblast integrin adhesion and signaling regulate mineralization, J. Dent. Res., 80 (2001), pp. 1540-1544,

[31] T. Traini, M. Degidi, R. Strocchi, S. Caputi, A. Piattelli, Collagen fiber orientation near dental implants in human bone: Do their organization reflect differences in loading?, Journal of Biomedical Materials Research Part B: Applied Biomaterials, 74B (2005), pp. 538-546, 10.1002/jbm.b.30245

[32] O. Andrukhov, R. Huber, B. Shi, S. Berner, X. Rausch-Fan, A. Moritz, N.D. Spencer, A. Schedle, Proliferation, behavior, and differentiation of osteoblasts on surfaces of different microroughness, Dent. Mater., 32 (2016), pp. 1374-1384, 10.1016/j.dental.2016.08.217

[33] H. Xie, M. Chua, I. Islam, R. Bentini, T. Cao, J.C. Viana-Gomes, A.H. Castro Neto, V. Rosa, CVD-grown monolayer graphene induces osteogenic but not odontoblastic differentiation of dental pulp stem cells, Dent. Mater., 33 (2016), pp. e13-e21, 10.1016/j.dental.2016.09.030

[34] M. Sartori, G. Giavaresi, A. Parrilli, A. Ferrari, N.N. Aldini, M. Morra, C. Cassinelli, D. Bollati, M. Fini, Collagen type I coating stimulates bone regeneration and osteointegration of titanium implants in the osteopenic rat, Int. Orthop., 39 (2015), pp. 2041-2052, 10.1007/s00264-015-2926-0 
[35] S. Stewart, D. Shea, C.P. Tarnowski, M.D. Morris, D. Wang, R. Franceschi, D.L. Lin, E. Keller, Trends in early mineralization of murine calvarial osteoblastic cultures: a Raman microscopic study, J. Raman Spectrosc., 33 (2002), pp. 536-543,

[36] W.E. Brown, J.P. Smith, J.R. Lehr, A.W. Frazier, Octacalcium Phosphate and Hydroxyapatite: Crystallographic and Chemical Relations between Octacalcium Phosphate and Hydroxyapatite, Nature, 196 (1962), pp. 1050-1055, 10.1038/1961050a0

[37] E.M. Czekanska, M.J. Stoddart, R.G. Richards, J.S. Hayes, In search of an osteoblast cell model for in vitro research, European Cells and Materials, 24 (2012), pp. 1-17, 10.22203/eCM.v024a01 


\section{Captions}
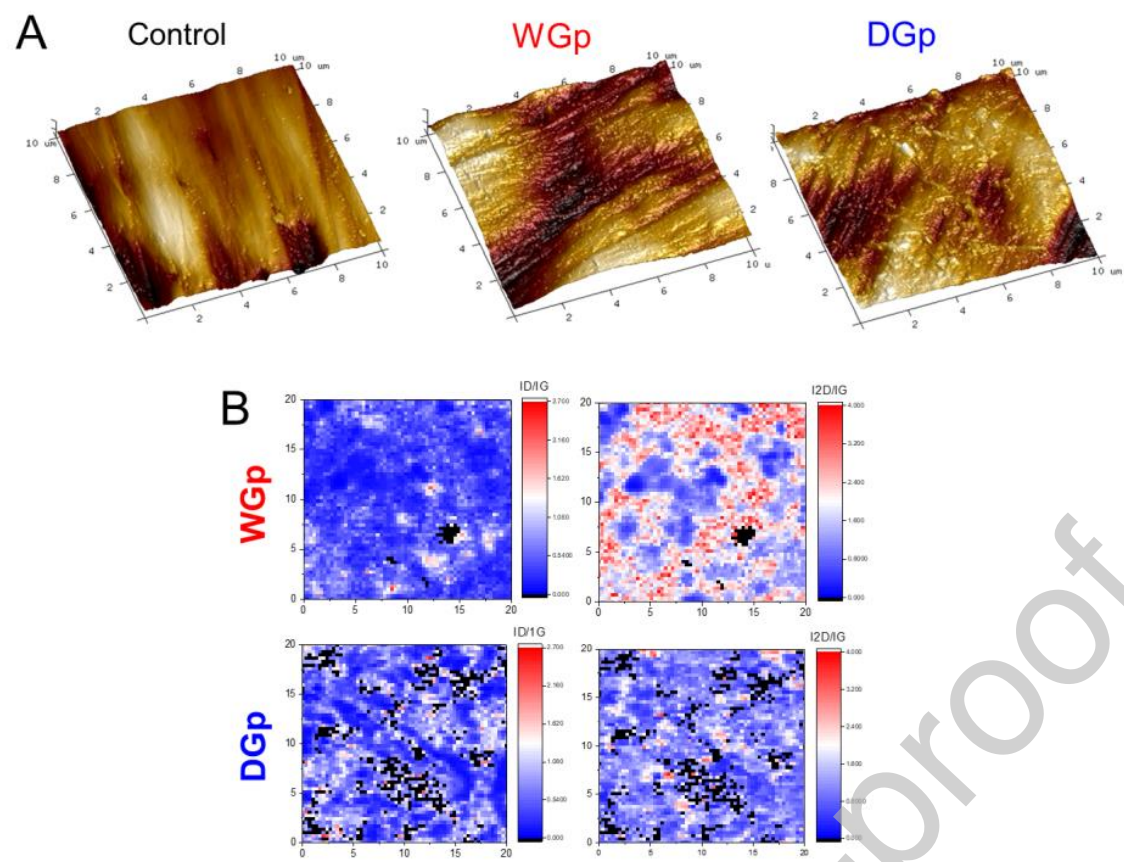

Fig. 1. AFM and Raman characterization of graphene coated titanium. (A) The titanium samples coated with graphene via wet (WGp) or dry (DGp) transfer techniques presented wrinkles on the surface.

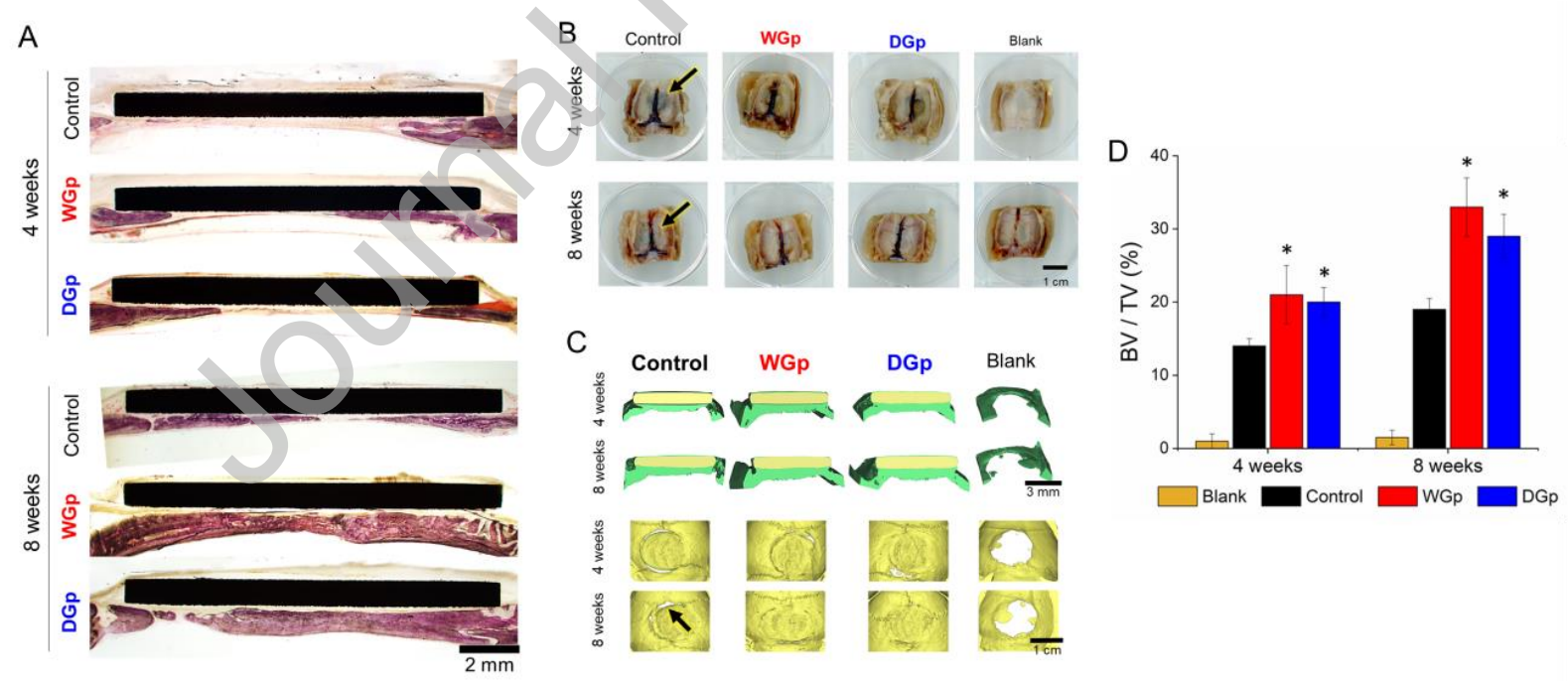

Fig. 2. Graphene coated titanium increased bone formation regardless of the transfer technique. (A) The histological analysis showed increased bone formation for both WGp and DGp at both time points evaluated. (B) The bone formation observed on WGp and DGp was thicker. Countor of WGp and DGp discs could not be recognized in the interior of the skull whereas the Control was idetifiable even after 8 weeks from implantation (arrows). ( $C$ and D) Healing of rat calvarial defects assessed by micro- 
computed tomography. The three-dimensional reconstructions from the $\mu \mathrm{CT}$ data show significantly higher bone regeneration for graphene coated titanium compared with the Control at 4 and 8 weeks from implantation. The percentage of bone volume/total volume (BV/TV) was similar for both WGp and DGp indicating that the transfer technique does affect graphene's osteogenic potential.

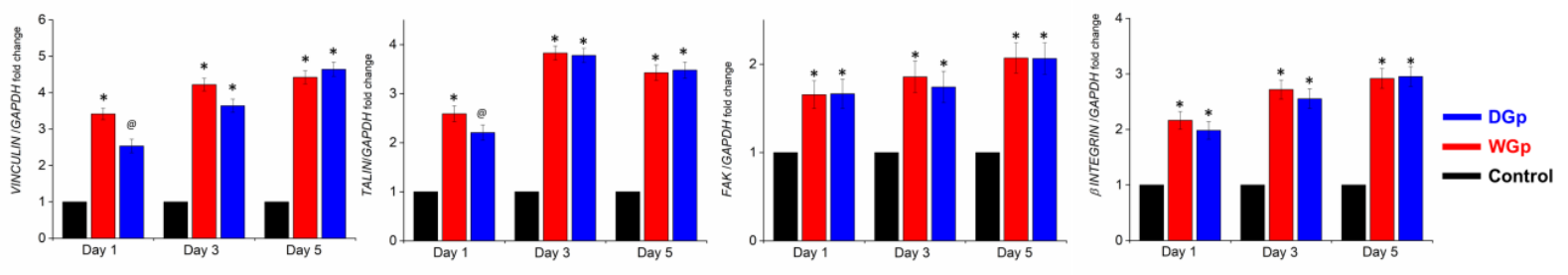

Fig. 3. The expression of all collagen genes tested were significantly higher on graphene coated samples compared to the Control at Day 3 and 5 (A to C). Cells cultured on WGp and DGp were able to produce higher amounts of collagen compared with those on the Control for all time points tested. (D) There were no differences in cell stiffness for none of groups studied at Day $5(*$ denotes statistical difference compared with the Control for that time point, $p<0.05$ ) .

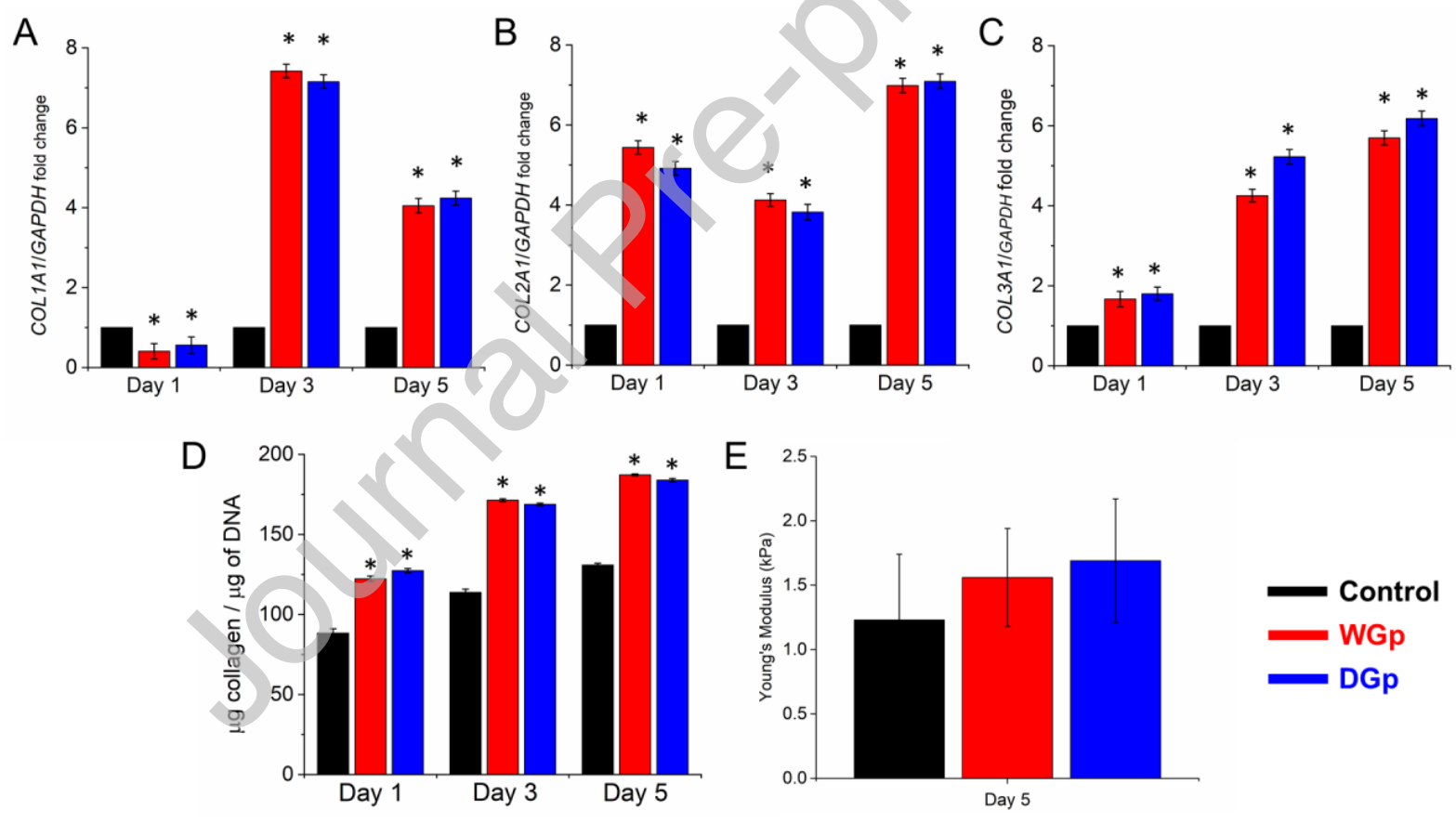

Fig. 4. Graphene coated samples by both techniques induced higher expression of genes related to cell adhesion and mechanosensing like VINCULIN, TALIN, FAK and $\beta$ INTEGRIN at all time points compared with the Control (* denotes statistical difference compared with the Control for that time point. ${ }^{@}$ denotes statistical difference compared with WGp for that time point, $p<0.05$ ). 

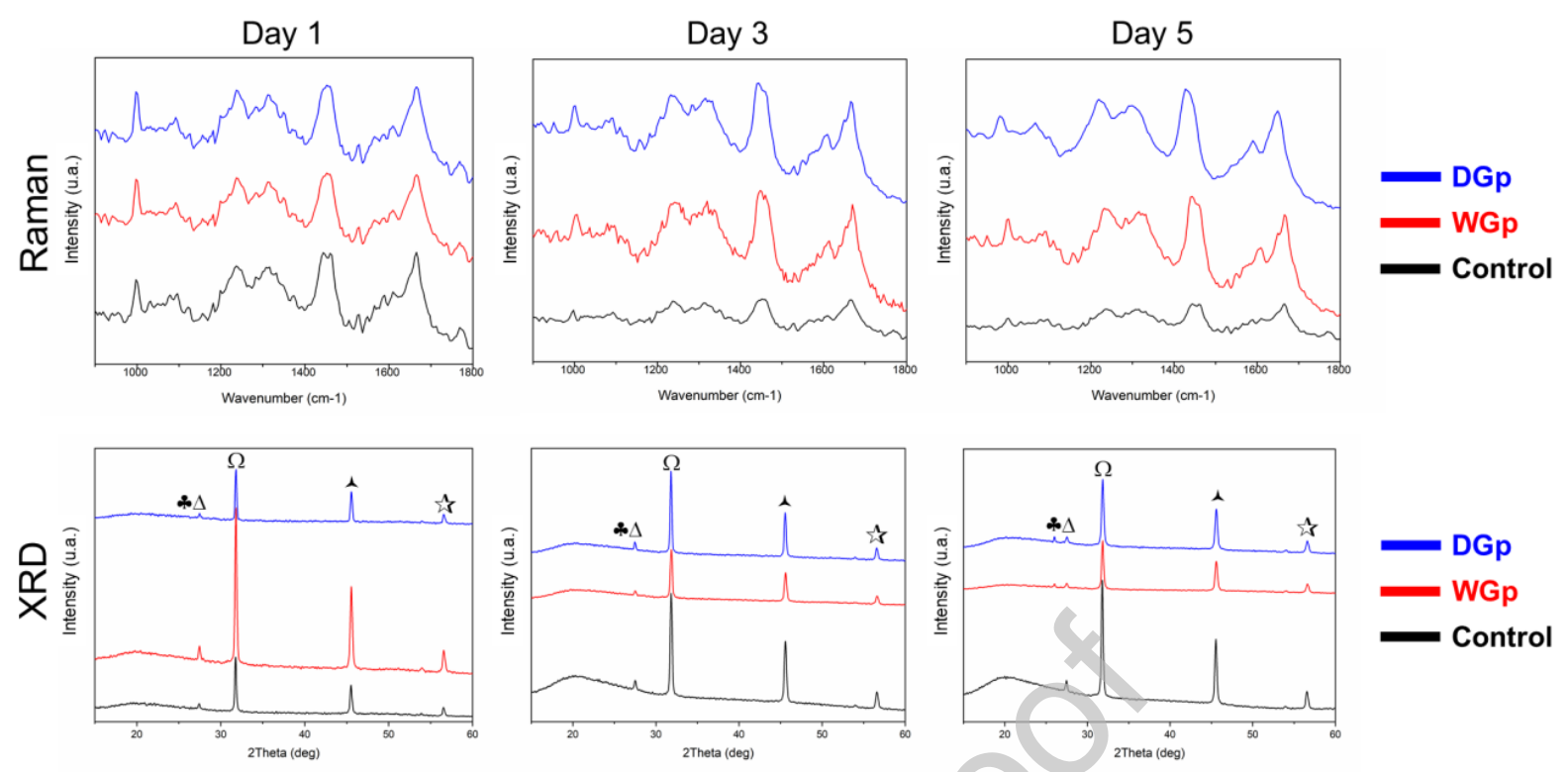

Fig. 5. Raman spectroscopy (top) was used to identify potential gross changes in the spectra related to cellular structure. There was no obvious changes in the signature peaks for cellular structures $\mathrm{CH}_{2}-\mathrm{Wag}$ $\left(1450 \mathrm{~cm}^{-1}\right)$, phosphate $\left(1030 \mathrm{~cm}^{-1}\right)$, amide I $\left(1660 \mathrm{~cm}^{-1}\right)$ and amide III (1239 and $\left.1318 \mathrm{~cm}^{-1}\right)$ regardless of the substrate and time point evaluated. Following, the mineralized deposits produced by MG-63 were characterized by XRD diffraction (bottom). The overall patterns were similar for all substrates and time points with a wide band suggestive of amorphous phase and peaks often related to mineralization processes. Both graphene coated samples presented peaks at $26^{\circ}$ suggestive of the presence of octacalcium phosphate (OCP). The trends were similar for both WGp and DGp suggesting similar potential to deposit mineralized structures $\left(*=\mathrm{OCP}, \Delta=\mathrm{ACP}, \Omega=\mathrm{Ca}^{2+}\right.$ deficient hydroxyapatite, $\lambda=\mathrm{TCP}$ and $\hat{\mathrm{s}}=$ DCPA). 
Table 1. The Raman characterization revelaed a total coverage yield of at least $90 \%$ for both techniques. The analyses of $\mathrm{I}_{\mathrm{D}} / \mathrm{I}_{\mathrm{G}}$ ratio demonstrates a similar defect distribution in WGp (0.47) and DGp (0.47 and 0.64 , respectively. Black pixels indicating absence of graphene, mappings scale in $\mu \mathrm{m}$ ).

\begin{tabular}{|l|l|l|l|l|}
\hline & Coverage area (\%) & FWHM & $\mathrm{I}_{2 \mathrm{D}} / \mathrm{I}_{\mathrm{G}}$ & $\mathrm{I}_{\mathrm{D}} / \mathrm{I}_{\mathrm{G}}$ \\
\hline WGp & 99.15 & 28.36 & 1.85 & 0.47 \\
\hline DGp & 90.87 & 34.74 & 1.51 & 0.64 \\
\hline
\end{tabular}

